\title{
Current Tropical Medicine Reports: A Path Forward to Highlight Research and Clinical Advances, New Trends and Innovations
}

\author{
Rojelio Mejia • Maria Elena Bottazzi
}

Published online: 29 December 2013

(C) Springer International Publishing AG 2014

Keywords Tropical medicine $\cdot$ Research $\cdot$ Clinical advances $\cdot$ Review articles $\cdot$ Infectious diseases, vaccine $\cdot$ Viruses ·

Parasites · Biosciences · Dengue virus · Virology ·

Bacteriology · Parasitology · Mycology · Entomology ·

Immunology $\cdot$ Cellular and molecular biology $\cdot$ Epidemiology

Welcome to the first edition of Current Tropical Medicine Reports! This is an exciting time in tropical medicine; the ongoing advancements in basic and applied science to clinical research have now positioned the discipline beyond what the giants in our field may have expected in their wildest dreams. Manson, Gorgas, and Kean would be impressed with the audacious milestones that the scientific community has completed: from having complete sequences of nematode genomes to new innovations for the development of vaccines and drugs against pathogens that have plagued mankind for eons.

The goal of this journal is to enhance the understanding of tropical medicine by delving into the latest advances in the wide expanse of the world of biosciences. This journal seeks to access a varied audience of readers, from the established principal investigators wanting to gain new ideas for basic or applied research to the first-year medicine resident or fellow wanting to learn more about these global diseases and apply

R. Mejia $\cdot$ M. E. Bottazzi $(\bowtie)$

Sabin Vaccine Institute and Texas Children's Hospital Center for

Vaccine Development, National School of Tropical Medicine, Baylor

College of Medicine, Houston, TX, USA

e-mail: Bottazzi@bcm.edu

R. Mejia

e-mail: rojelio.mejia@bcm.edu that knowledge to the care of their patients. New issues will be released on a quarterly basis, and will focus on current highlights, hot topics, and a review of recent advances in the study of pathogens that affect morbidity and mortality worldwide. The topics will include virology, bacteriology, parasitology, mycology, entomology, immunology, cellular and molecular biology, epidemiology, and more; the journal will address the latest in diagnostics, treatment, and prevention of these global diseases and pathogens.

To add variety, the quarterly selections will include one or more organisms from a pathogenesis group and describe the latest preventive and therapeutic strategies, evolving trends, and important developments in their burden of disease, the geographical distribution, and advances in research \& development, all the way to clinical trials and clinical management. The articles will focus on work from the past three years and the advancements that are shaping the way we study and practice medicine.

The section editors and authors have been selected from a cadre of scientists and physicians who are leaders in their field. They bring years of accumulated knowledge and research, but also their expert opinion and personal experience in their field. In addition the journal has compiled a diverse group of global leaders to participate as the editorial board.

This inaugural edition covers viral tropical medicine with a focus on Dengue virus, from emergence in the Americas to the regulation of its replication. Also included is a review of the helminth Toxocara, from development of molecular assays to clinical presentations of toxocariasis.

As recently assessed in the Global Burden of Disease Study 2010, tropical diseases continue to have huge contributions towards the world's morbidity (1). Furthermore, the notion that tropical medicine is limited to tropical climates or resource-limited areas in developing countries is now changing. Active transmission of these 
diseases is being identified in the G20 countries and countries with large economies, especially in areas where poor populations reside (2). Therefore, the misconception that health care providers should not have to worry about such "exotic" diseases is no longer valid. In reality, the world is filled with millions of species throughout all geographies, climates and terrains. Furthermore, there are now very few places that are remote and not visited. The ability to travel to most places and return to their country of origin in less than 24 hours, which is generally faster than most latency periods of these pathogens, allows for relatively asymptomatic patients to be able to return from their travels with these tropical diseases.

We invite the reader to come along in this educational and scientific endeavor to join the giants and rising stars in a field that has global implications.

\section{Compliance with Ethics Guidelines}

Conflict of Interest Rojelio Mejia and Maria Elena Bottazzi declare that they have no conflict of interest.

Human and Animal Rights and Informed Consent This article does not contain any studies with human or animal subjects performed by any of the authors.

\section{References}

1. Murray CJ, Vos T, Lozano R, Naghavi M, Flaxman AD, Michaud C, et al. Disability-Adjusted Life Years (Dalys) for 291 Diseases and Injuries in 21 Regions, 1990-2010: A Systematic Analysis for the Global Burden of Disease Study 2010. Lancet. 2012;380(9859):2197223. EPUB 2012/12/19.

2. Hotez PJ. The Disease Next Door. Foreign Policy. 2013. Epub March 26,2013 